\title{
Baryons in the unquenched quark model
}

\author{
R. Bijker*, S. Díaz-Gómez*, M.A. Lopez-Ruiz ${ }^{\dagger}$ and E. Santopinto** \\ *Instituto de Ciencias Nucleares, Universidad Nacional Autónoma de México, AP 70-543, 04510 \\ Mexico DF, Mexico \\ ${ }^{\dagger}$ Physics Department and Center for Exploration of Energy and Matter, Indiana University, \\ Bloomington, IN 47408, USA \\ ${ }^{* *}$ Istituto Nazionale di Fisica Nucleare, Sezione di Genova, via Dodecaneso 33, I-16146 Italy
}

\begin{abstract}
In this contribution, we present the unquenched quark model as an extension of the constituent quark model that includes the effects of sea quarks via a ${ }^{3} P_{0}$ quark-antiquark paircreation mechanism. Particular attention is paid to the spin and flavor content of the proton, magnetic moments and $\beta$ decays of octet baryons.
\end{abstract}

Keywords: Phenomenological quark models, light quarks, protons and neutrons

PACS: $12.39 .-\mathrm{x}, 14.65 . \mathrm{Bt}, 14.20 . \mathrm{Dh}$

\section{INTRODUCTION}

In the constituent quark model (CQM) hadrons are described as a system of constituent (or valence) quarks and antiquarks, $q q q$ for baryons and $q \bar{q}$ for mesons. Despite the success of the quark model, there is strong evidence for the existence of exotic degrees of freedom (other than valence quarks) in hadrons from CQM studies of electromagnetic and strong couplings of baryons that are, on average, underpredicted by CQMs [1]. More direct evidence for the importance of quark-antiquark components in the proton comes from measurements of the $\bar{d} / \bar{u}$ asymmetry in the nucleon sea [2,3] and the proton spin crisis [4]. The pion cloud in the nucleon holds the key to understand the flavor asymmetry and the spin-crisis of the proton [2, 3, 5]. Moreover, angular momentum conservation of the pionic fluctuations of the nucleon leads to a relation between the flavor asymmetry and the contribution of orbital angular momentum to the spin of the proton $\mathscr{A}(p)=\Delta L[6]$.

The aim of this contribution is to discuss the role of valence and sea quarks in the nucleon in the framework of a simplified version of the unquenched quark model (UQM) in which only the effects of the pion cloud is taken into account. It is shown that the pion cloud offers a qualitative understanding of the results obtained in previous numerical studies [7], and thus provides important insights into the properties of the nucleon.

\section{UNQUENCHED QUARK MODEL}

The unquenched quark model developed in [7] is motivated by earlier studies on extensions of the quark model in which the $q \bar{q}$ pairs are created in the ${ }^{3} P_{0}$ state with the quantum numbers of the vacuum $[8,9]$. The present approach is based on a CQM to which the quark-antiquark pairs are added as a perturbation, employing a ${ }^{3} P_{0}$ model 
for the $q \bar{q}$ pair creation. The pair-creation mechanism is inserted at the quark level and the one-loop diagrams are calculated by summing over the intermediate baryon-meson states (see Fig. 1).

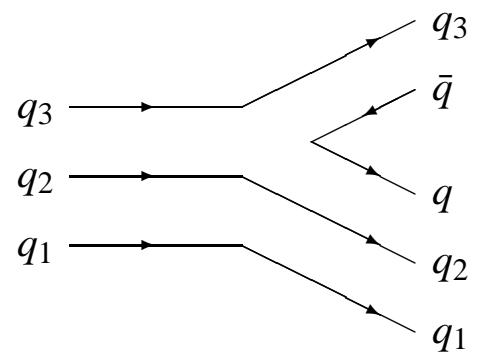

FIGURE 1. Schematic quark line diagram for $A \rightarrow B C$.

Under these assumptions, the baryon wave function consists of a zeroth order threequark configuration $|A\rangle$ plus a sum over higher Fock components due to the creation of quark-antiquark pairs. The resulting baryon wave function is given by [7]

$$
\left|\psi_{A}\right\rangle=\mathscr{N}\left[|A\rangle+\sum_{B C l J} \int d \vec{K} k^{2} d k|B C, l, J ; \vec{K}, k\rangle \frac{\left\langle B C, l, J ; \vec{K}, k\left|T^{\dagger}\right| A\right\rangle}{\Delta E_{B C}(k)}\right]
$$

where $\Delta E_{B C}(k)=M_{A}-E_{B}(k)-E_{C}(k)$ is the energy difference calculated in the rest frame of the initial baryon $A$. The operator $T^{\dagger}$ creates a quark-antiquark pair in the ${ }^{3} P_{0}$ state with the quantum numbers of the vacuum: $L=S=1$ and $J=0$. The ${ }^{3} P_{0}$ transition amplitude can be expressed as [10]

$$
\left\langle B C, l, J ; \vec{K}, k\left|T^{\dagger}\right| A\right\rangle=\delta(\vec{K}) M_{A \rightarrow B C}(k)
$$

where $\delta(\vec{K})$ is a consequence of momentum conservation in the rest frame of A.

\section{Flavor and spin content}

In this contribution, we employ a simplified version of the UQM in which only the contribution of the pion cloud is taken into account. Table 1 shows the results for the flavor and spin content of the proton. In the UQM, the coefficients appearing in Table 1 are expressed in terms of integrals over the relative momentum $k$

$$
\begin{aligned}
a^{2} & \rightarrow \int k^{2} d k \frac{\left|M_{N \rightarrow N \pi}(k)\right|^{2}}{\Delta E_{N \pi}^{2}(k)}, \\
b^{2} & \rightarrow \int k^{2} d k \frac{\left|M_{N \rightarrow \Delta \pi}(k)\right|^{2}}{\Delta E_{\Delta \pi}^{2}(k)}, \\
2 a b & \rightarrow \int k^{2} d k \frac{M_{N \rightarrow N \pi}^{*}(k) M_{N \rightarrow \Delta \pi}(k)+M_{N \rightarrow \Delta \pi}^{*}(k) M_{N \rightarrow N \pi}(k)}{\Delta E_{N \pi}(k) \Delta E_{\Delta \pi}(k)},
\end{aligned}
$$


TABLE 1. Spin and flavor content of the proton in he constituyent quark model (CQM) and the unquenched quark model (UQM), normalized to the flavor asymmetry using the E866/NuSea value [11] (UQM1) and using the NMC value [12] (UQM2).

\begin{tabular}{cccccc}
\hline & CQM & UQM & UQM1 & UQM2 & Exp \\
\hline $\mathscr{A}(p)=\Delta L$ & 0 & $\frac{2 a^{2}-b^{2}}{3\left(1+a^{2}+b^{2}\right)}$ & $* 0.118$ & $* 0.158$ & $0.118 \pm 0.012$ \\
$\Delta u$ & $\frac{4}{3}$ & $\frac{4}{3}-\frac{38 a^{2}+b^{2}-16 a b \sqrt{2}}{27\left(1+a^{2}+b^{2}\right)}$ & 1.132 & 1.064 & $0.842 \pm 0.013$ \\
$\Delta d$ & $-\frac{1}{3}$ & $-\frac{1}{3}+\frac{2 a^{2}+19 b^{2}-16 a b \sqrt{2}}{27\left(1+a^{2}+b^{2}\right)}$ & -0.368 & -0.380 & $-0.427 \pm 0.013$ \\
$\Delta s$ & 0 & 0 & 0.000 & 0.000 & $-0.085 \pm 0.018$ \\
$\Delta \Sigma=\Delta u+\Delta d+\Delta s$ & 1 & $1-\frac{4 a^{2}-2 b^{2}}{3\left(1+a^{2}+b^{2}\right)}$ & 0.764 & 0.684 & $0.330 \pm 0.039$ \\
$g_{A}=\Delta u-\Delta d$ & $\frac{5}{3}$ & $\frac{5}{3}-\frac{40 a^{2}+20 b^{2}-32 a b \sqrt{2}}{27\left(1+a^{2}+b^{2}\right)}$ & 1.500 & 1.444 & $1.2723 \pm 0.0023$ \\
\hline
\end{tabular}

which only depend on the ${ }^{3} P_{0}$ coupling strength. The results for the UQM in Table 1 are also valid for the meson-cloud model in which the coefficients $a$ and $b$ multiply the $N \pi$ and $\Delta \pi$ components of the nucleon wave function [6]. The $a b$ term denotes the contribution from the cross terms between the $N \pi$ and $\Delta \pi$ components. In the UQM, the value of the cross term $a b$ is not equal to the product of $a$ and $b$, although the numerical values are close. Since the UQM contains the full spin and isospin structure, it satisfies the relation between the flavor asymmetry and the contribution of the orbital angular momentum to the spin of the proton $\mathscr{A}(p)=\Delta L[6]$, and therefore $\Delta \Sigma=1-2 \Delta L$. In the absence of the pion cloud $\left(a^{2}=b^{2}=2 a b=0\right)$ we recover the results of the CQM.

The results for the spin and flavor content of the proton are normalized to the proton flavor asymmetry. The fourth column is normalized to the E866/NuSea value [11], and the fifth column to the somewhat higher NMC value [12]. The experimental values of the spin content were obtained by the HERMES [13] and the COMPASS [14] Collaborations. Table 1 shows the results from the HERMES Collaboration.

\section{Magnetic moments}

The magnetic moments of the octet baryons constitute one of the early successes of the constituent quark model. Hence, for any extension of the quark model, it is important to verify whether the good agreement of the CQM is maintained. Table 2 shows that this is indeed the case for the unquenched quark model. Just as for the CQM, the quark magnetic moments are fitted to the magnetic moments of the proton, neutron and $\Lambda$ hyperon. 
TABLE 2. Magnetic moments. The experimental values are taken from Ref. [15].

\begin{tabular}{crrrc}
\hline & CQM & UQM1 & UQM2 & Exp \\
\hline$p$ & 2.793 & 2.793 & 2.793 & 2.793 \\
$n$ & -1.913 & -1.913 & -1.913 & -1.913 \\
$\Lambda$ & -0.613 & -0.613 & -0.613 & $-0.613 \pm 0.004$ \\
\hline$\Sigma^{+}$ & 2.673 & 2.589 & 2.509 & $2.458 \pm 0.010$ \\
$\Sigma^{0}$ & 0.791 & 0.783 & 0.751 & \\
$\Sigma^{-}$ & -1.091 & -1.023 & -1.007 & $-1.160 \pm 0.025$ \\
$\Xi^{0}$ & -1.435 & -1.359 & -1.290 & $-1.250 \pm 0.014$ \\
$\Xi^{-}$ & -0.493 & -0.530 & -0.552 & $-0.651 \pm 0.003$ \\
$\Sigma^{0} / \Lambda$ & 1.630 & 1.640 & -1.638 & $1.61 \pm 0.08$ \\
\hline
\end{tabular}

\section{Fluctuations}

In the UQM, it is straightforward to calculate the fluctuation probabilities. The probability that a proton fluctuates in $n \pi^{+}$is given by

$$
\left|\left\langle n \pi^{+} \mid p\right\rangle\right|^{2}=\frac{2 a^{2}}{3\left(1+a^{2}+b^{2}\right)},
$$

whereas the total probability for a pion fluctuation of the proton is given by

$$
|\langle N \pi \mid p\rangle|^{2}+|\langle\Delta \pi \mid p\rangle|^{2}=\frac{a^{2}+b^{2}}{1+a^{2}+b^{2}} .
$$

The numerical results are shown in Table 3. The UQM1 values are in good agreement with the experimental values as determined in an analysis of forward neutron production in electron-proton collisions by the H1 and ZEUS Collaborations at DESY [16, 17], and in a study of the quark distribution functions measured in Drell-Yan experiments and semi-inclusive DIS experiments [18]. The UQM2 values are about $30 \%$ higher than the UQM1 values.

TABLE 3. Pion fluctuations of the proton.

\begin{tabular}{ccccc}
\hline & UQM1 & UQM2 & Exp & Ref \\
\hline$\left|\left\langle n \pi^{+} \mid p\right\rangle\right|^{2}$ & 0.180 & 0.241 & $0.17 \pm 0.01$ & {$[16,17]$} \\
$|\langle N \pi \mid p\rangle|^{2}+|\langle\Delta \pi \mid p\rangle|^{2}$ & 0.455 & 0.609 & 0.470 & {$[18]$} \\
\hline
\end{tabular}

\section{SUMMARY AND CONCLUSIONS}

In this contribution, we studied the properties of the proton in the framework of the unquenched quark model in which the ${ }^{3} P_{0}$ coupling strength was normalized to the ob- 
served value of the proton flavor asymmetry. It was shown that whereas the pion fluctuations maintain the good results of the constituent quark model for the magnetic moments, they help to understand the discrepancies between the CQM and the experimental data. Their inclusion leads to a reduction of quark model value of $\Delta u$ and $g_{A}$, and give rise to a sizeable contribution (25-30\%) of orbital angular momentum to the spin of the proton. In addition, it was found that the probabilities for pion fluctuations in the UQM are in good agreement with the values determined in analyses of the available experimental data.

\section{ACKNOWLEDGMENTS}

This work was supported in part by grant IN107314 from DGAPA-PAPIIT, Mexico.

\section{REFERENCES}

1. S. Capstick and W. Roberts, Prog. Part. Nucl. Phys. 45, 241 (2000).

2. S. Kumano, Phys. Rep. 303, 183 (1998).

3. G.T. Garvey and J.C. Peng, Prog. Part. Nucl. Phys. 47, 203 (2001).

4. A.W. Thomas, Int. J. Mod. Phys. E 18, 1116 (2009);

S.E. Kuhn, J.P. Chen and E. Leader, Progr. Part. Nucl. Phys. 63, 1 (2009).

5. J. Speth and A.W. Thomas, Adv. Nucl. Phys. 24, 83 (1998).

6. G.T. Garvey, Phys. Rev. C 81, 055212 (2010).

7. R. Bijker and E. Santopinto, Phys. Rev. C 80, 065210 (2009) [arXiv:0912.4494];

E. Santopinto and R. Bijker, Phys. Rev. C 82, 062202(R) (2010).

8. N.A. Törnqvist, Acta Phys. Polon. B 16, 503 (1985);

P. Zenczykowski, Ann. Phys. (N.Y.) 169, 453 (1986).

9. P. Geiger and N. Isgur, Phys. Rev. D 55, 299 (1997).

10. W. Roberts and B. Silvestre-Brac, Few-Body Systems 11, 171 (1992).

11. R.S. Towell et al. (FNAL E866/NuSea Collaboration), Phys. Rev. D 64, 052002 (2001).

12. M. Arneodo et al. (NMC Collaboration), Nucl. Phys. B 487, 3 (1997).

13. A. Airapetian et al. (HERMES Collaboration), Phys. Rev. D 75, 012007 (2007).

14. V. Yu. Alexakhin et al. (COMPASS Collaboration), Phys. Lett. B 647, 8 (2007).

15. K.A. Olive et al. (Particle Data Group), Chin. Phys. C 38, 090001 (2014).

16. A. Bunyatyan and B. Povh, Eur. Phys. J. A 27, 359 (2006).

17. B. Povh and M. Rosina, Bled Workshops in Physics 12, 82 (2011).

18. W.-C. Chang and J.-C, Peng, Phys. Rev. Lett. 106, 252002 (2011). 